\title{
IR Sensors Array for Robots Localization Using K Means Clustering Algorithm
}

\author{
Israa S. AL-Furati $^{1}$, Abdulmuttalib T. Rashid ${ }^{2}$, Alaa Al-Ibadi ${ }^{3}$ \\ 1,2 Electrical Engineering Department, University of Basrah, Basrah Iraq. \\ ${ }^{3}$ School of Computing, Science and Engineering, University of Salford, Salford, UK. \\ israa.subri.1@email.com, abdturky@email.com, a.f.a.al-ibadi@edu.salford.ac.uk
}

\begin{abstract}
IR receiver sensors distributed uniformly in the environment. the position of a multi-robot system in an indoor localization system is successfully estimated using a new algorithm. The necessary information for the localization development is collected by scanning the IR sensor array in the environment. The scanning process is done column by column to recognize and state the position of the IR receiver's sensors, which receive signals from the IR transmitter that is fixed on the robot. This principle of scanning helps to minimize the required time for robot localization. The $k-$ means clustering algorithm is used to estimate the multi-robot's locations by isolating the labeled IR receivers into clusters. Basically, the multi-robot position is estimated to be in the middle of each cluster. Simulation results demonstrate the advanced algorithm in estimation to the multi-robot positions for various dimensional IR receiver's array.
\end{abstract}

Keywords - k-means clustering algorithm, Localization, IR sensors.

\section{INTRODUCTION}

Due to a fast increase in data and multimedia services, the lack of localization and path planning have significantly increased [1], especially in indoor areas, such as exhibition rooms, libraries, garages, supermarkets, airport lobbies, underground parking, etc. However, the faultless localization technique has not been applied due to the limitations of localization timing, complex indoor environments, and positioning accuracy. Usually a GPS [2] is the well-known technology used to detect a position in the localization field, but it is not suitable in an indoor environment due to various obstacles in the line of sight, such as walls, human beings and equipment, as well as the propagation effect of electromagnetic waves that lead to multi-path action [3]. A particular characteristic of this system is that the suggested algorithm incorporates the k-means clustering algorithm to estimate the multi-robot position of indoor environments by evaluating the signal from the IR receiver sensor of every column in a combined sender-receiver fashion, taking the information about the position of the sender into account.

Specifically, the present work can be summarized by the following points:

- The localization problem is solved by using an array of IR receiver sensors distributed uniformly in the environment.

- The necessary information for the localization process is collected by scanning the IR sensor array in the environment.

- The scanning process is done column by column in order to recognize and label the IR receivers, which receive signals from the IR transmitter that the robot is equipped with.
- The robot's position is estimated by isolating the labeled IR receivers into clusters using the k-means clustering algorithm.

- The middle of each cluster in the multi-robot system represents the location of one robot.

The utilization of an IR-system is very accurate and also easily carried by someone because they are light-weight and the IR (transmitter and receiver) sensors are small. The system does not require a lot of time for connection and conservation. This paper is rearranged as follows: Section (II) describes the indoor multi-robot localization system. In section (III) the specifics about the planned algorithm of kmeans clustering are explained. Simulation parameters and comparative results are presented in section (IV). Finally, conclusions are conferred in section (V).

\section{RELATED WORK}

The localization field was technologically advanced by suggesting a good indoor position system (IPS) using many technologies [4] like ultrasound, (WLAN), radio-frequency identification (RFID), (IR) sensors TX \& RX sensor networks, Bluetooth, ultra-wideband (UWB), vision analysis, magnetic signals and audible sound. These services extended the IPS field in companies, universities and research areas. Infrared (IR) localization systems are a promising technology. It is applied with wired or wireless devices, such as mobile phones, TV's, PDAs, printers, etc. [5]. In the 1990s, the first indoor localization systems at AT\&T Cambridge were fitted with symbolic location information to every active badge, such as the area where the active badge is and an area in a building. Meanwhile, in a smart construction of the Active Badge system, they employed a spread IR technology in order to verify the sensing location [6]. The IR indoor localization system, based on a Wiimote 
controller, was integrated with its sensors in robot space, even though it is limited to a 1-dimentional axis network which is unable to aggregate the robot environment for the dynamic system [7]. However, when a big indoor environment is being used, then the two IR landmarks are located within the camera view and the information about the robot task can be done efficiently [8]. It does not automatically locate the landmarks and it takes a long time until localization is acquired. A passive IR sensing system mainly depends on estimating the location of people in a small room but with more complexity interconnection networks [9]. The IR sensor also maps the openings in walls with indoor detection, as well as monitoring the doors and windows of vehicles and buildings [10]. Conversely, the IR location system has drawbacks, which are the problems with privacy and security. Moreover, the IR signals have some limitations for sensing locations, like interference from sunlight and fluorescent light [11]. The problem can be solved by using electronic and optical filters to diffuse the trouble from the light sources [12] and accomplish a signal processing with noise cancelling algorithms at the receivers, but increasing the cost of the localization system [11].

\section{THE INDOOR ROBOTS' LOCALIZATION SYSTEM}

The presented work introduces a new algorithm for the indoor multi-robot localization system based on IR sensors. The IR LEDs sensors are divided into two categories: the first one is characterized by four IR transmitters fitted onto each robot, and the second one is characterized by a matrix of different numbers of IR receivers distributed uniformly in the environment $((16 * 16),(32 * 32)$ and $(64 * 64)$ IR receiver sensors). Fig. 1 shows $\left(16^{*} 16\right)$ an empty-hole environment. The central unit scans the IR receiver sensor in an array column by column to recognize an IR transmitter signal within its sensing range. Only IR receiver sensors within the sensing range of the IR transmitters are labelled. These labelled IR sensors are distinguished as a group of IR receiver sensors. In order to estimate the multi-robot places the k-means clustering algorithm is applied to isolate the located IR receivers into clusters. The results obtained have clearly demonstrated that the center of each cluster represents the location of one robot when using the proposed method.

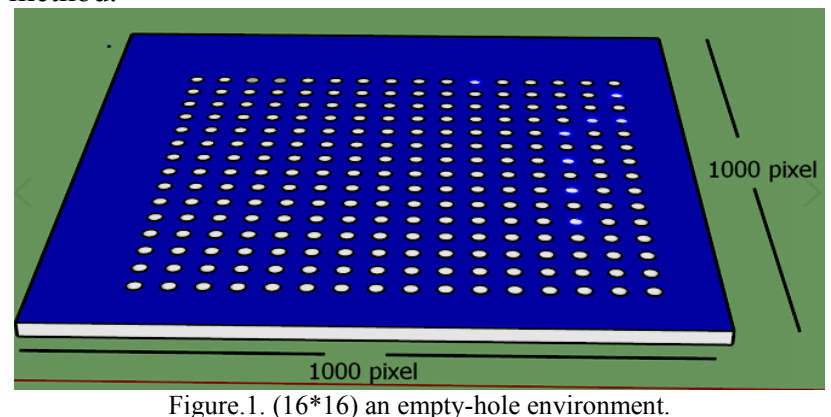

Figure.1. (16*16) an empty-hole environment.

\section{A. The IR receiver sensors array}

The proposed indoor environment consists of a twodimensional environment with multiple holes distributed uniformly and filled with an array of IR receiver sensors. The suggested localization system consists of multi-robots as shown in Fig.2.a. The IR transmitter sensors of this system are represented by four IR transmitters fixed on each robot as shown in Fig.2.b. These IR transmitters are always in transmitting status. The organized axis of each IR receiver sensor is artificial, to be known by the control unit in the system. The multi-robot localization process is done by scanning the IR receiver in an array column by column to identify which one senses any one of the IR transmitters equipped with robots. The sensed IR receivers are labelled one by one and distinguished by a yellow color, as shown in Fig.2.c.

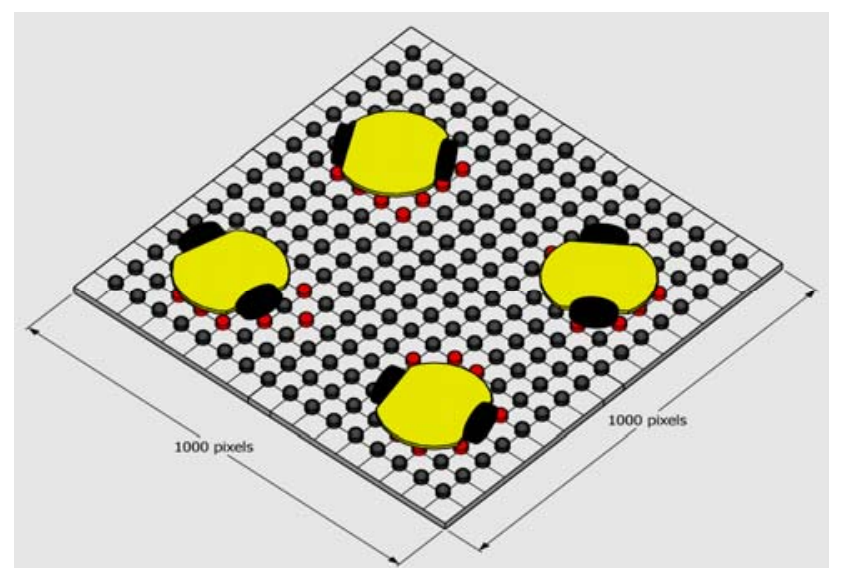

(a)

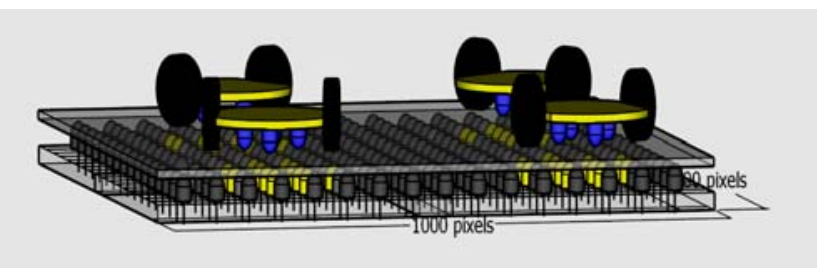

(b)

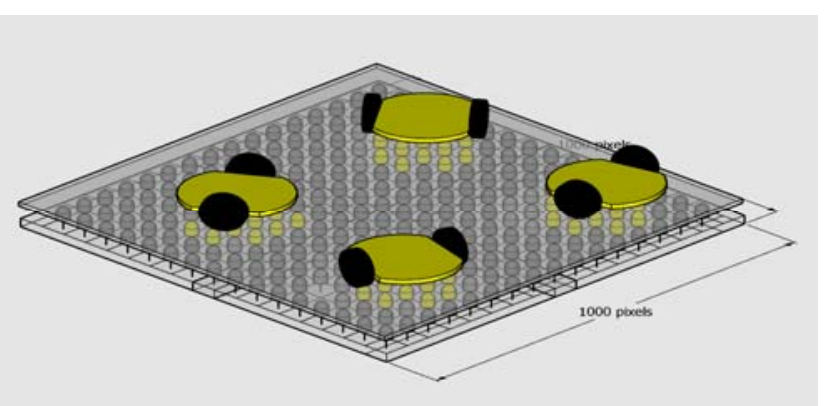

(c)

Figure.2. The indoor localization environment. (a) $\left(16^{*} 16\right)$ IR receiver's environment with 4 robots. (b) 4 robots each one equiped with 4 IR transmitter (blue IR LED). (c) The final vision environement. 


\section{K-MEANS CLUSTERING ALGORITHM}

The k-means clustering algorithm was advanced by J.A. Hartigan and M.A.Wong at Yale University as a subdividing procedure [13]. It is a simple and wonderful way to split the datasets because it can be used with a large set of data. Working with a big database requires an information clustering technique that is a very important tool which requires no prior knowledge about how it is distributed. The hierarchical clustering method is another clustering technique but, in comparison with the first algorithm, it is simple in implementation and requires little memory and computational efficiency [14]. Working with this algorithm helps to identify how many clusters there are in the database and if this information is unavailable. Multiple trials must be prepared to find the best number of clusters. In this paper the aim of the k-means technique is to partition the labelled IR sensors into $\mathrm{k}$ clusters (each IR sensor added to a cluster with a lower Euclidean distance). Fig.3. shows the suggested flow chart for working the k-means clustering.

The procedure is:

1) Randomly select $\mathrm{k}$ points in the environment to be the initial positions of the $\mathrm{k}$ clusters.

2) Distribute the labelled IR sensors into groups. The principle of distribution is achieved by measuring the Euclidean distances ED (equation 1) between each labeled IR receiver sensor and all the chosen $k$ points.

$$
E D=\sqrt{\sum_{i=1}^{I L}\left(C_{i}-x_{i}\right)^{2}}
$$

Where $\mathrm{n}$ is the total number of the labelled IR sensors, $\mathrm{xi}$ is the related node $\mathrm{i}$ (one of the characterized IR sensors) and the cluster $\mathrm{i}$ location is $\mathrm{Ci}$. Each group of IR receivers are constructed by adding each IR receiver sensor to one of the $\mathrm{k}$ points (which has minimum ED from this IR sensor).

3) Every individual IR receiver sensor LED is treated as one cluster. The centroid point of each cluster is computed by using equation 2 .

$$
\text { Centroid point } i=\left(\frac{\left(\sum_{i=1}^{m} x_{i}\right)}{m}, \frac{\left(\sum_{i=1}^{m} y_{i}\right)}{m}\right)
$$

Where $m$ is the number of IR receiver sensors in cluster $i$.

4) Make the $k$ centroid points the new positions of the cluster groups and repeat step 2 and 3 to redistribute the IR receiver sensors among these groups depending on the ED distances among the IR receiver sensors and the centroid points.

5) Repeat steps 2 to 4 until there are no IR receiver sensor LEDs added to each group. (Cluster $\mathrm{K}$, must be listed).

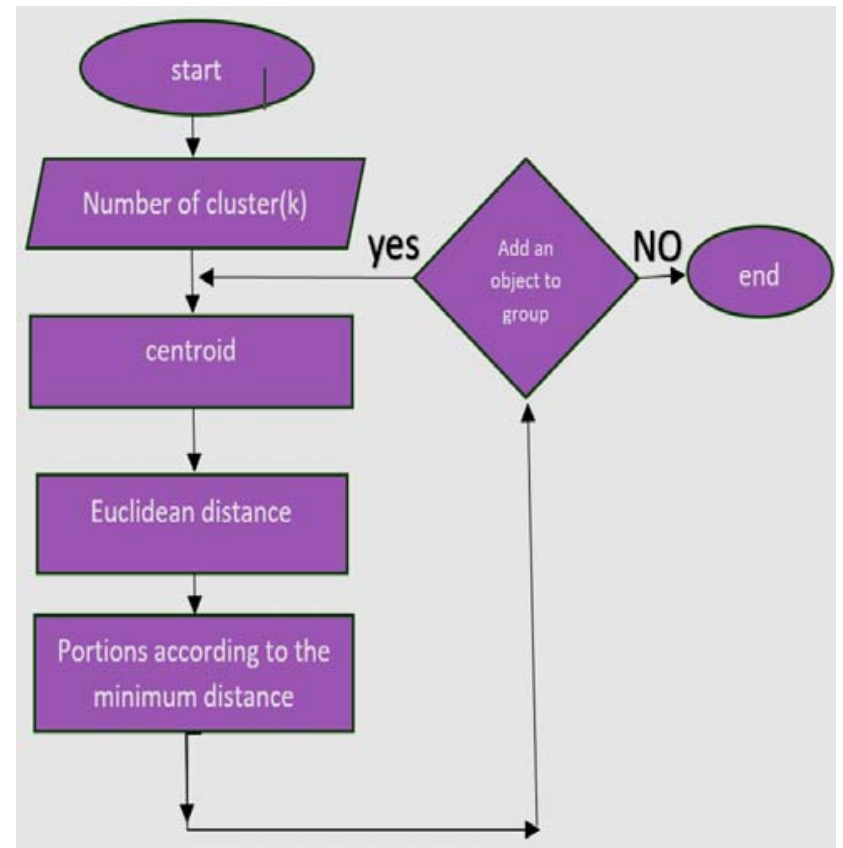

Figure.3. Flow chart for the k-means clustering algorithm

This algorithm is converging for similar measurements. During the first few iterations, most of the IR receiver sensors are convergence [15]. Fig.4. shows the stages of executing the algorithm on individual labels of IR sensors.

\section{SIMULATION RESULTS}

A new indoor multi-robot localization system is validated using some actual simulations. These simulations are performed with multi-robots; each one has 4 IR transmitters. The simulations are repeated for 50 different topologies representing various robot positions, for different sensing ranges for the IR receiver sensor nodes and for different distances between the IR receiver sensor nodes. The dimensions of the environment are assumed to be $(1000 * 1000)$ pixels with $(16 * 16,32 * 32$ and $64 * 64)$ IR receiver sensors which are distributed uniformly. The overall parameters used in this system simulation are:

1) The number of IR receiver sensors fitted to the environment.

2) The position change to every robot.

3) The maximum sensing range of the IR receiver sensor nodes.

Fig.5.a, b and c. demonstrate the first cluster of simulations for the localization system on a robot 1, 2, 3 and 4 , with a sensing range equal to 60 pixels in some environments. The best result, i.e. the minimum error, is obtained under dimensions with $(64 * 64)$ pixels. The first simulation is implemented in a multi-robot with 4 IR transmitters and it is repeated for a different sensing range of this sensor. 
Fig.6. demonstrates the simulation result collected by repeating the first simulation 50 times, with changed locations of the robots, to achieve the relationship between the operational percentage of the k-mean clustering and the changed values of the sensing range. The result confirms that to obtain a full sensing range you need 80,40 and 20 pixels in the environment of $(16 * 16),(32 * 32)$ and $(64 * 64)$, IR receiver sensors respectively. The result confirms that to obtain a full sensing range you need 80 pixels in all cases.

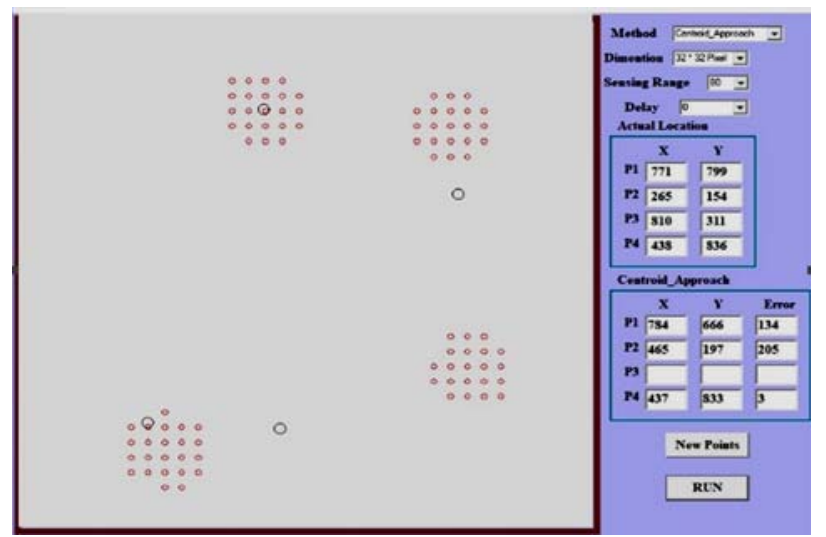

(a)

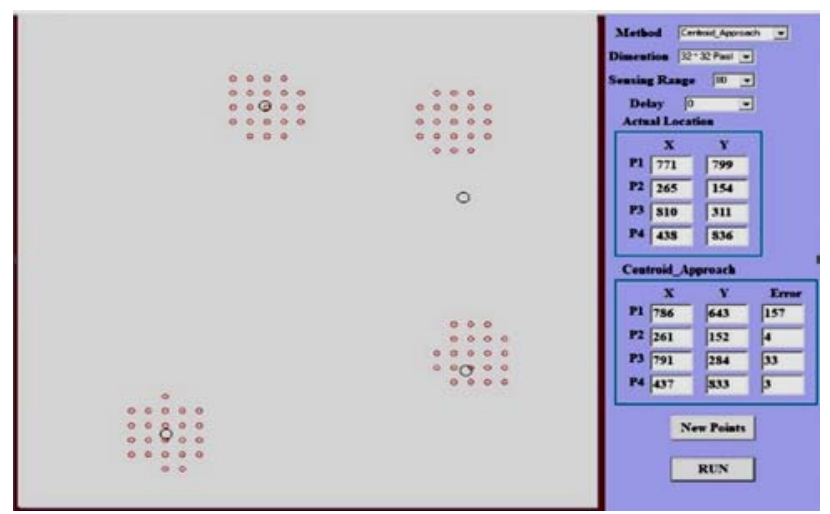

(b)

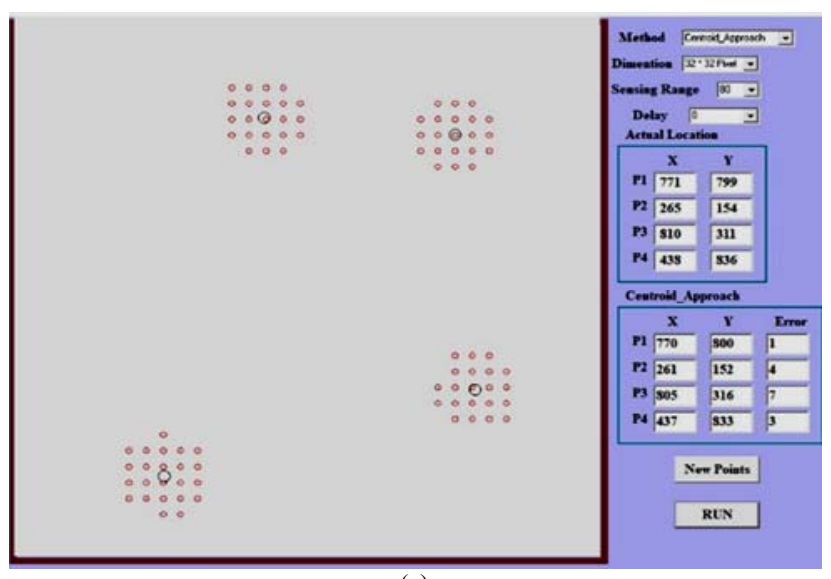

(c)

Figure.4. several iterations to reach the center of the robot.

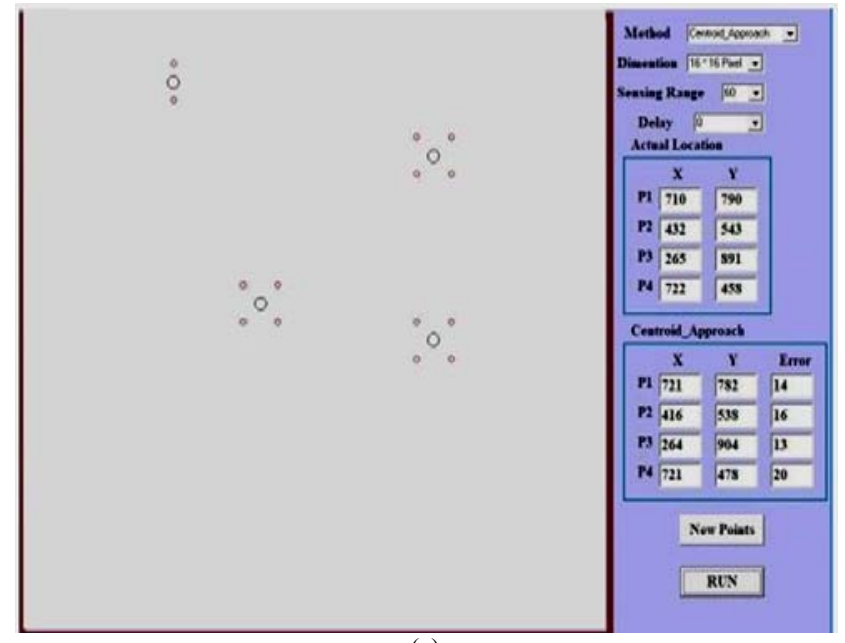

(a)

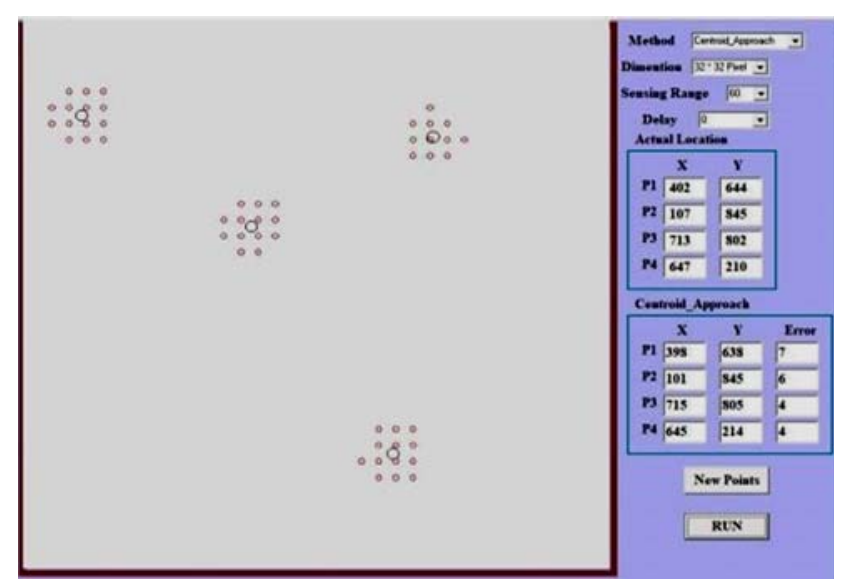

(b)

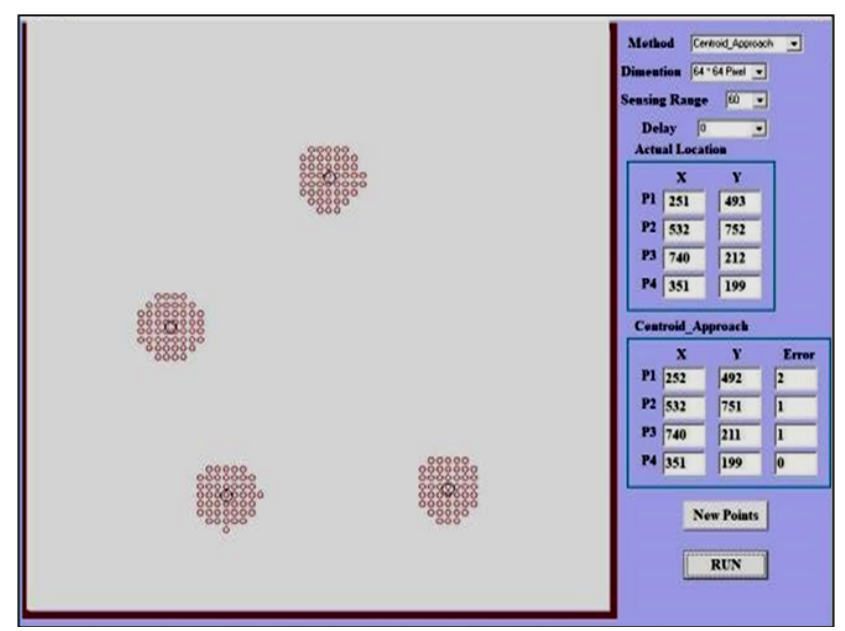

(c)

Figure.5. Simulation of environment for localization the robot 1,2,3 and 4 with sensing range $=60$ pixels. (a) $\left(16^{*} 16\right)$ environement. (b) $(32 * 32)$ environment. (c) $(64 * 64)$ environment. 


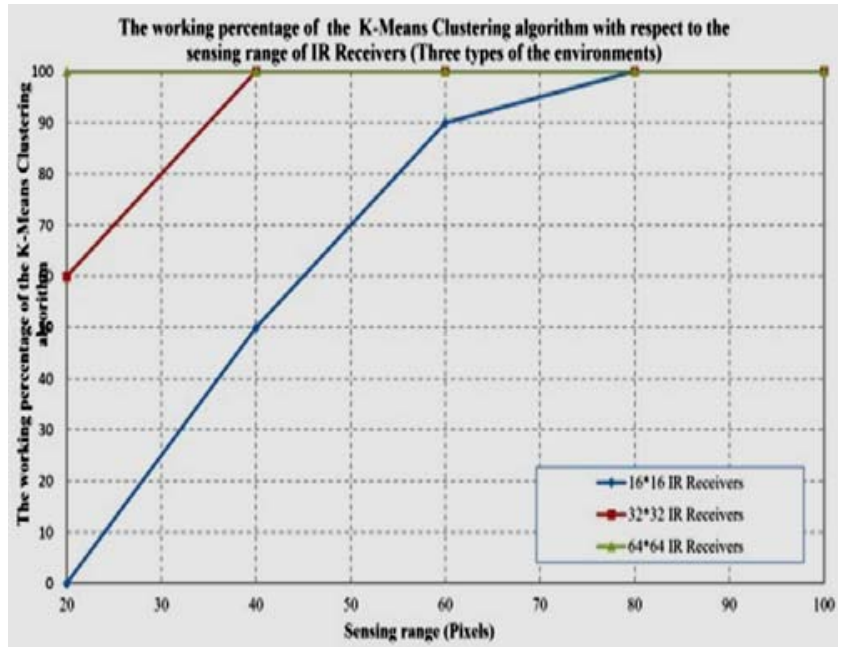

Figure.6.the percentage working of the k-means clustering algorithm with respect to the IR receivers (three types of environment)

The second group of the simulation is implemented for comparing the average of the iterations of the k-mean clustering with respect to the IR receiver sensing range (Fig. 7). By repeating this simulation 50 times, the result shows that as the sensing range of the IR receiver sensor is increased, the average repeated iteration numbers also increase.

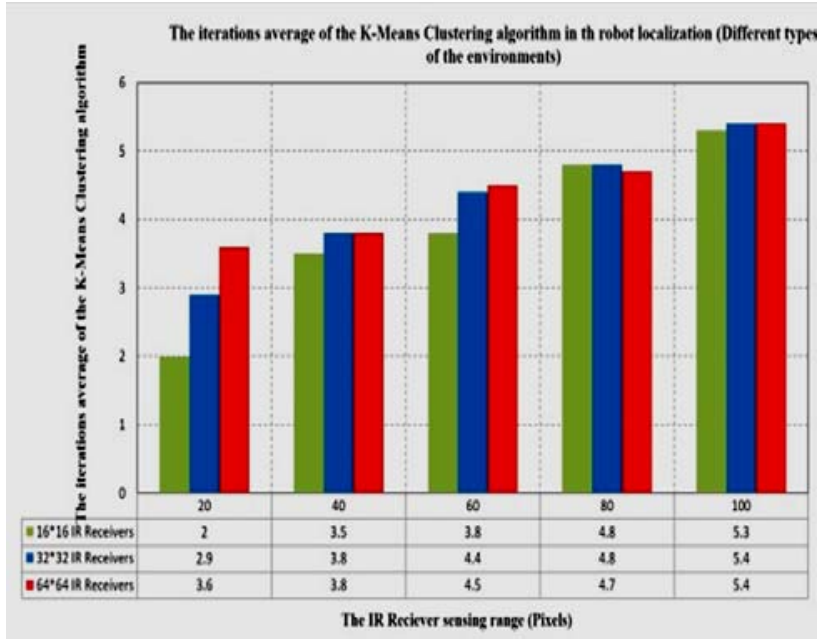

Figure.7.the average iteration of the k-means clustering algorithm in robot localization with different environments.

The same comparison procedure in Fig.7 is repeated for the multi-robot localization (Fig. 8). This comparison indicates that the average error is reduced when using the (64*64) IR receivers LEDs environment.

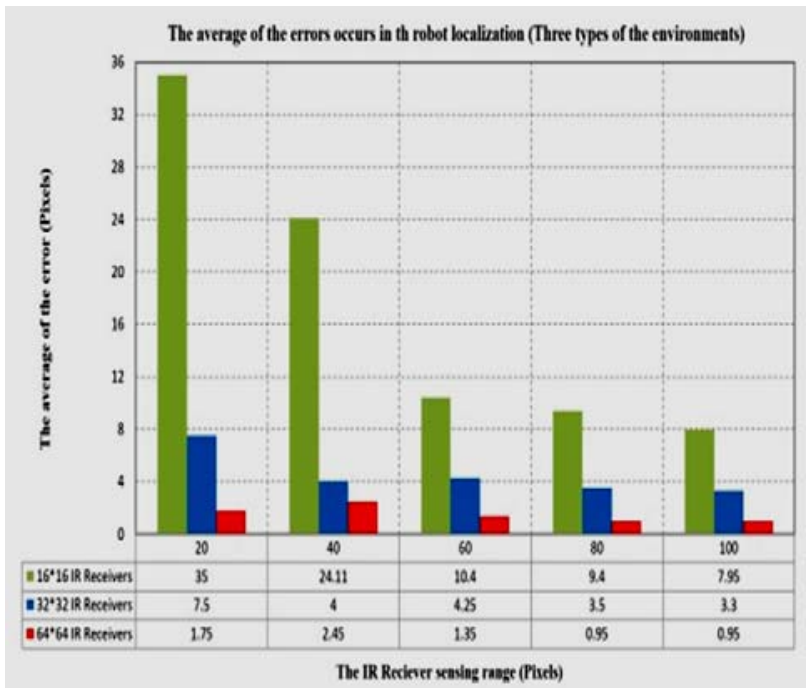

Fig.8. the average of the errors occurs in the robot localization.

\section{CONCLUSION}

An indoor robot localization system is validated. The problem of localization is solved by using an array of IR receiver sensors that are distributed uniformly in the environment. The development of localization is executed and tested on the four-robot system with various numbers of environments and different sensing ranges. A full detection range is reached by using an environment of $(64 * 64)$ IR receiver LEDs sensor and the results confirm that the greatest sensing range is 20 pixels. Similarly, the result shows that the average number of errors that occur in the robot localization is reduced as the IR receiver sensing range increases. Moreover, the best case is obtained when the $(64 * 64)$ IR receiver sensors are used with a sensing range equal to 20 pixels.

\section{REFERENCES}

[1] Y. GU, A .Lo, S. Member, and I. Niemegeers," A Survey of Indoor Positioning Systems for Wireless Personal Networks", IEEE Communications Surveys \& Tutorials, Vol. 11, No. 1, pp. $13-32$, 2009.

[2] B. Hofmann-Wellenhof, H. Lichtenegger, and J. Collins, " Global Positioning Systems: Theory and Practice," Springer, 5 editions, 2001.

[3] A. M. Ladd, K.E. Bekris, A. P. Rudys, D. S. Wallach, and L. E. Kavraki," On the Feasibility of Using Wireless Ethernet for Indoor Localization", IEEE Transactions on Robotics and Automation, Vol. 20, No. 3.

[4] I.S. Alfurati and A. T. Rashid," Performance Comparison of Three Types of Sensor Matrices for Indoor Multi-Robot Localization", International Journal of Computer Applications (0975 - 8887) Volume 181 - No. 26, pp. 22-29, 2018.

[5] O. A. Hasan, A. T. Rashid and R. S. Ali (2016). A Hybrid approach for multi-node localization and Identification. Basra Journal of Engineering Sciences, 16-(2), 11- 20.

[6] R. WANT, A. HOPPER, V. FALC $\sim \mathrm{O}$ and J. GIBBONS," The Active Badge Location System", Practice and Experience. 
[7] P.-W.Chen, K.-S. OU and K-S. Chen "IR Indoor Localization and Wireless Transmission for Motion Control in Smart Building Applications based on Wiimote Technology", SICE Annual Conference 2010 August 18-21, 2010, The Grand Hotel, Taipei, Taiwan.

[8] B.Sohn, J. Lee, H.Chae, and W. Yu ," Localization System for Mobile Robot Using Wireless Communication with IR Landmark ",Korea MIC and IITA through IT leading R\&D Support Program (2005-S092-03, USN-based Ubiquitous Robotic Space Technology Development).

[9] K. Hashimoto, T. Tsuruta, K. Morinaka, N.Yoshiike ," High performance human information sensor", Sensors and Actuators 79 2000 46-52, ELSEVIER.

[10] A.M. Flynn," Combining Sonar and Infrared Sensors for Mobile Robot Navigation", The International Journal of Robotics Research, RMIT UNIVERSITY on May 28, 201.

[11] X. N. Fernando, S. Krishnan, H. Sun and K. K-Moud," Adaptive denoising at Infrared wireless receivers", The International Society for Optical Engineering · September 2003.

[12] C. Lee, Y. Chang, G. Park, J. Ryu, S.-G. Jeong, S. Park, J. W.Park, "H. C. Lees and K-s. Hong," Indoor Positioning System Based on Incident Angles of Infrared Emitters", The 30th Annular Conference of the IEEE Industrial Electronics Society, November 2 -6, 2004.

[13] NCSS Statistical Software," K-Means Clustering”, NICSS.COM.

[14] L. Morissette and S. Chartier," The k-means clustering technique: General considerations and implementation in Mathematica", Tutorials in Quantitative Methods for Psychology, Vol. 9, No.1, pp $15-24,2013$.

[15] Z. Zhang," K-means Algorithm g Cluster Analysis in Data Mining”, $3 / 22 / 2012$. 